\title{
Stage III Vulvar Cancer AJCC v8
}

National Cancer Institute

\section{Source}

National Cancer Institute. Stage III Vulvar Cancer A/CC v8. NCI Thesaurus. Code C139623.

Stage III includes: T1-T2, N1-N2c, M0. T1: Tumor confined to the vulva and/or perineum. Multifocal lesions should be designated as such. The largest lesion or the lesion with the greatest depth of invasion will be the target lesion identified to address the highest PT stage. Depth of invasion is defined as the measurement of the tumor from the epithelial-stromal junction of the adjacent most superficial dermal papilla to the deepest point of invasion. T2: Tumor of any size with extension to adjacent perineal structures (lower/distal third of the urethra, lower/distal third of the vagina, anal involvement). N1: Regional lymph node metastasis with one or two lymph node metastases each less than $5 \mathrm{~mm}$, or one lymph node metastasis equal to $5 \mathrm{~mm}$. N2a: Three or more lymph node metastases each less than $5 \mathrm{~mm}$. It includes micrometastasis, N2mi. N2b: Two or more lymph node metastases equal to $5 \mathrm{~mm}$. N2c: Lymph node(s) metastasis with extranodal extension. M0: No distant metastasis. (AJCC 8th Ed.) 